\title{
A woman with a red eye from a carotid-cavernous sinus fistula
}

\author{
Jonathan A. Micieli MD CM, Harleen Bedi MD, Timo Krings MD
}

Cite as: CMAJ 2017 January 23;189:E113-5. doi: 10.1503/cmaj.151029

A 57-year-old woman presented to the emergency department with a left red eye of five months' duration. A dull ache had recently developed behind that eye, but the patient had not experienced discharge, visual changes or pruritus. She had no history of trauma, headache or other neurologic symptoms.

The patient reported that during the first week after her eye became red, she had seen an optometrist. She had been referred to an ophthalmologist, who diagnosed allergic conjunctivitis and prescribed daily administration of olopatadine eye drops. The patient was seen in follow-up two weeks later, at which time she was encouraged to continue the prescribed eye drops. Her symptoms persisted, and she visited an emergency department three months later, where an ophthalmologist was consulted. She was started on a low-potency steroid eye drop (loteprednol etabonate $0.5 \%$ four times daily) and was told to return to the original ophthalmologist for follow-up. Because her symptoms persisted, she came to our emergency department two weeks later for a second opinion.

On examination, the patient's best corrected visual acuity was 20/20 in the right eye and 20/25 in the left eye. Her pupils were equal in size and reactive to light without a relative afferent pupillary defect. The intraocular pressure was $10 \mathrm{~mm} \mathrm{Hg}$ in the right eye and $17 \mathrm{~mm} \mathrm{Hg}$ in the left eye. Extraocular movements were full, and there was $2 \mathrm{~mm}$ of left proptosis. The appearance of her eyes on presentation is shown in Figure 1. A dilated fundus examination was normal.

Given the presence of tortuous corkscrew episcleral vessels, chemosis (i.e., edema of the conjunctiva), asymmetric intraocular pressure and proptosis, we were concerned that the patient might have an anteriorly draining dural arteriovenous fistula or indirect carotidcavernous sinus fistula. Computed tomography (CT) angiography confirmed this diagnosis: specifically, there was early filling of the left cavernous sinus, left superior ophthalmic vein and left sphenoparietal sinus in the arterial phase of the scan (Figure 2). There was also reflux toward a partially thrombosed inferior petrosal sinus.

The patient underwent cerebral angiography for further assessment of the fistula (Figure 3), and the diagnosis of a Borden type II dural arteriovenous fistula was confirmed. Given the increased risk of intracranial hemorrhage from cortical venous reflux, embolization of the left dural carotid-cavernous sinus fistula was performed with a transvenous approach. A microcathether was advanced into the appropriate compartment inside the cavernous sinus, and coiling was performed. Subsequent control runs showed absence of reflux to the cortical veins and absence of early filling of the cavern-

\section{KEY POINTS}

- The presence of discharge (characteristic of conjunctivitis), vision changes and pain are important factors to consider when evaluating a patient with a red eye.

- A carotid-cavernous sinus fistula should be considered in a patient with chronic red eye (present longer than four weeks).

- Clinical features of a carotid-cavernous sinus fistula include tortuous corkscrew episcleral vessels, chemosis, asymmetric intraocular pressure, proptosis and an orbital bruit.

- Permanent loss of vision and intracranial hemorrhage may result if there is a delay in diagnosis and treatment of a dural arteriovenous fistula.

ous sinus (Figure 4). Postprocedural ophthalmologic assessment showed a reduction in dilatation of the episcleral vessels and chemosis; visual function remained normal.

\section{Discussion}

Red eye is commonly reported to primary care and eye care professionals. ${ }^{1}$ It may represent a benign, self-limited condition or, more rarely, may be the presenting sign of an intracranial process, as in this case. The presence of pain, visual changes, discharge and recent eye surgery are important factors to determine on historytaking, as explained in an evidence-based review. ${ }^{1}$ The unusual features that led us to suspect a carotid-cavernous sinus fistula were the extended duration of the problem, the presence of corkscrew

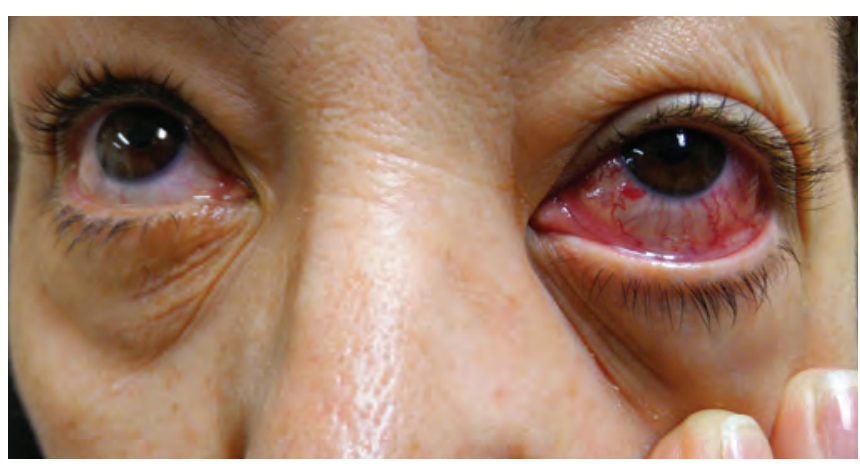

Figure 1: External examination of the left eye of a 57-year-old woman showing tortuous episcleral blood vessels and chemosis. The patient had proptosis, and intraocular pressure was greater in the left eye than the right eye. 
episcleral vessels rather than diffuse conjunctival redness, mild proptosis and asymmetric intraocular pressure. ${ }^{2}$ The absence of discharge and pain also eliminated some of the more common causes of red eye, such as conjunctivitis, iritis and scleritis. ${ }^{1}$

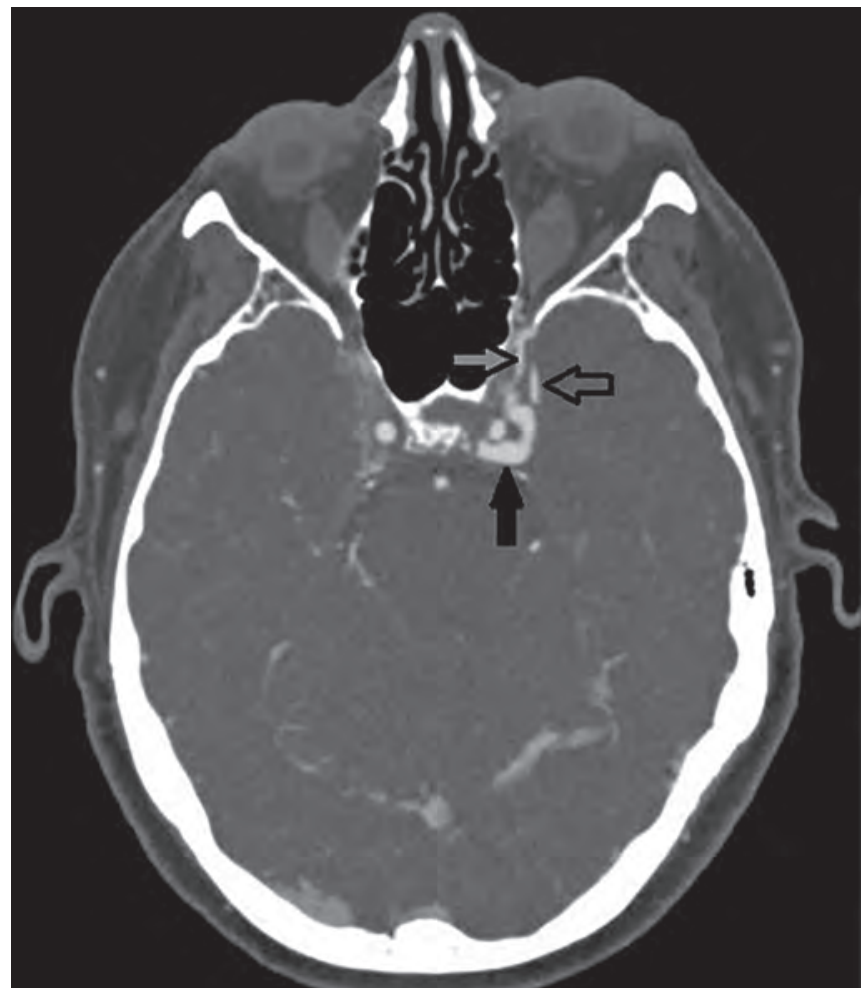

Figure 2: Arterial computed tomography angiography showing cortical venous reflux, with filling of the cavernous sinus (solid black arrow) and the superior ophthalmic vein anteriorly (darker grey arrow), and reflux toward the sphenoparietal sinus (lighter grey arrow).
A carotid-cavernous sinus fistula represents an abnormal communication between branches of the internal or external carotid arteries and the cavernous sinus. The symptoms and signs,_including corkscrew episcleral vessels, proptosis, asymmetric intraocular pressure and chemosis, are related to arterialization of the conjunctival and episcleral vessels and the superior ophthalmic vein and subsequent venous congestion. ${ }^{2}$ Pulsatile exophthalmos and an ocular bruit also support this diagnosis. ${ }^{2}$

The reason for development of dural carotid-cavernous sinus fistulas is still controversial. ${ }^{2}$ One hypothesis is that such fistulas develop spontaneously after rupture of one or more thin-walled dural arteries that normally traverse the cavernous sinus. ${ }^{3}$ After rupture, preformed dural arterial anastomoses not directly involved in the fistula dilate and contribute to the collateral blood supply, which results in an angiographic appearance indistinguishable from that of a congenital vascular malformation. A second theory claims that dural carotid-cavernous sinus fistulas develop in response to spontaneous venous thrombosis in the cavernous sinus and represent an attempt to provide a pathway for collateral venous outflow. ${ }^{4}$ This theory has the advantage of explaining the pathogenesis of arteriovenous fistulas that develop in the sigmoid and other dural sinuses. ${ }^{4}$

\section{Loss of vision}

As in this case, intraocular pressure may be asymmetrically elevated in patients with anteriorly draining dural carotid-cavernous sinus fistulas because of elevated episcleral venous pressure. The conventional outflow of the aqueous humour depends on the episcleral venous pressure. It is thought that for every $1 \mathrm{~mm} \mathrm{Hg}$ increase in episcleral venous pressure, there is an increase of at least $1 \mathrm{~mm} \mathrm{Hg}$ in intraocular pressure. ${ }^{5}$ Glaucomatous optic nerve damage, which is irreversible, may be seen in patients with chronically elevated intraocular pressure. In addition to glaucoma, these

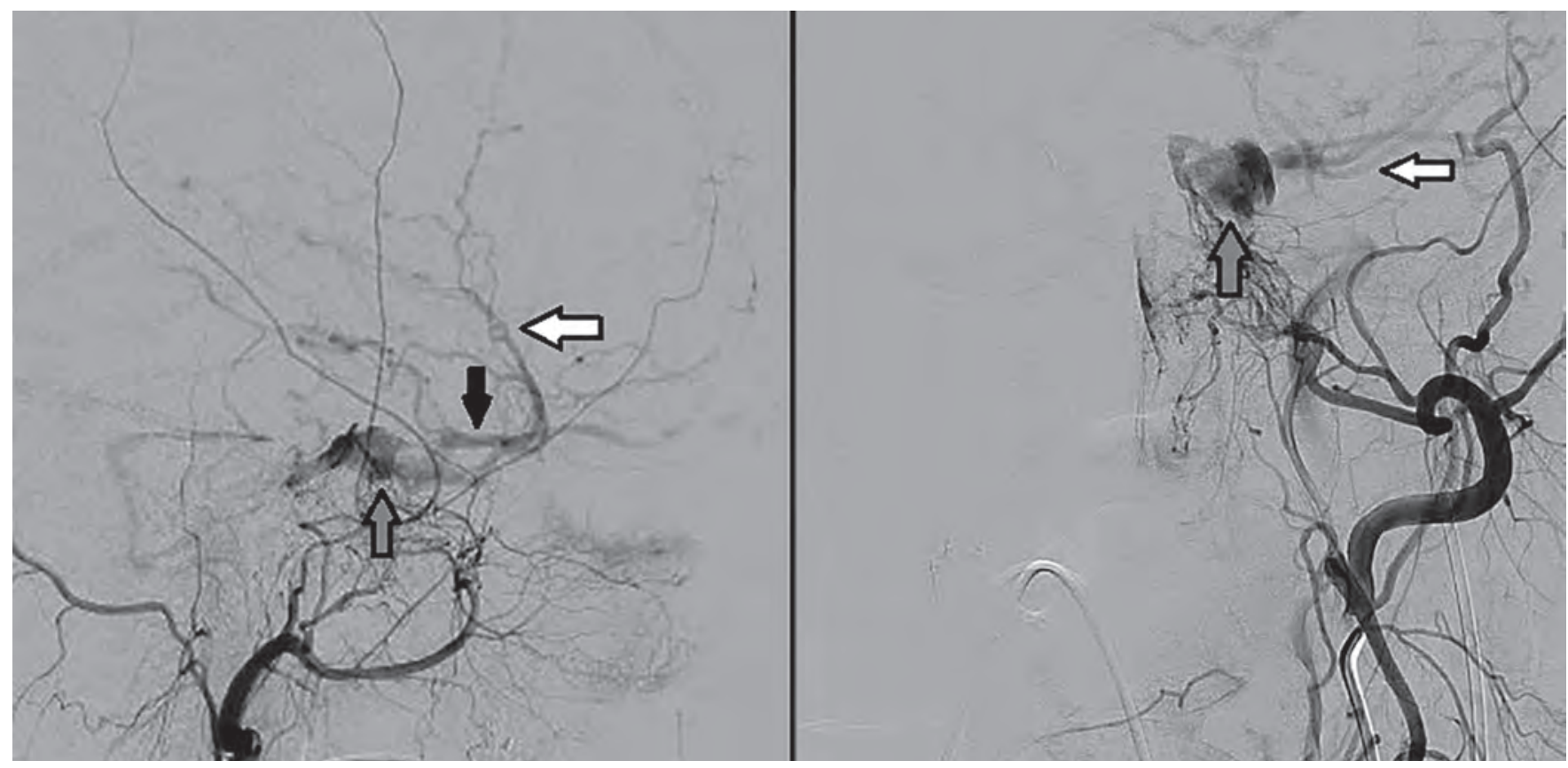

Figure 3: Lateral (left) and anteroposterior (right) cerebral angiography, with injection of contrast agent into the external carotid artery, showing cortical venous reflux, with filling of the cavernous sinus (solid grey arrow) and the ophthalmic vein anteriorly (solid black arrow), and reflux toward the sphenoparietal sinus (solid white arrow). 


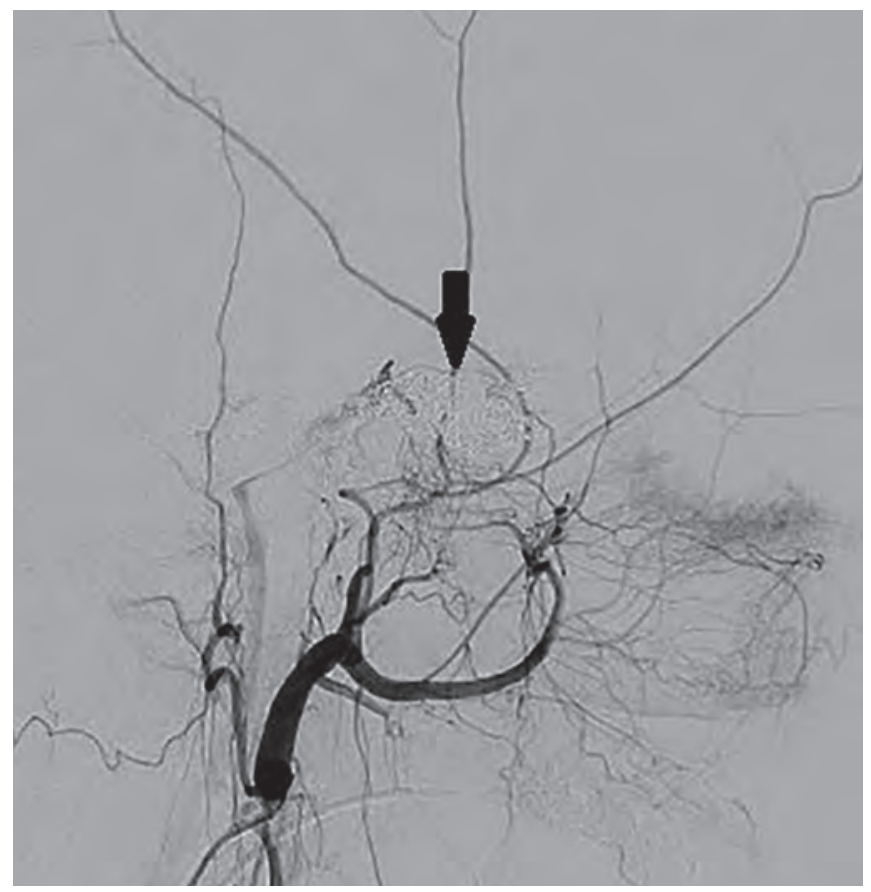

Figure 4: Post-treatment lateral cerebral angiography, with injection of contrast agent into the external carotid artery, showing complete occlusion of the fistulous point of the left carotid-cavernous fistula, with absence of early filling of the cavernous sinus (solid black arrow).

patients may experience permanent loss of vision through occlusion of the retinal vein or ischemic optic neuropathy. ${ }^{2}$

Carotid-cavernous sinus fistulas that drain posteriorly into the inferior and superior petrosal sinus generally present not with orbital symptoms (e.g., proptosis, chemosis, lid edema), but rather with "white-eyed" painful diplopia. ${ }^{2}$ The oculomotor nerve is most commonly involved, but palsy of the fourth and sixth nerves is also frequently seen. Vision loss may occur with posteriorly draining fistulas, but less commonly than is the case with anteriorly draining fistulas. ${ }^{2}$

\section{Management}

The presence of the characteristic features of carotid-cavernous sinus fistulas in a patient with red eye should prompt neuroimaging that includes angiography. ${ }^{6}$ Cerebral angiography is the gold standard for definitive diagnosis, classification and planning of treatment, because CT angiography may not show small feeding arteries or the exact site of fistulous communication. ${ }^{6}$

Certain low-flow dural carotid-cavernous sinus fistulas may close spontaneously. ${ }^{6}$ However, expert opinion suggests that patients with neurologic symptoms or cortical venous drainage on neuroimaging studies are at higher risk of intracranial hemorrhage and should be considered for closure on an urgent basis. ${ }^{6,7}$

Borden and colleagues ${ }^{7}$ proposed classification into three types of dural arteriovenous fistulas: type I, with antegrade drainage toward the heart via a dural sinus; type II, with drainage to a dural sinus and reflux into cortical veins; and type III, with drainage to the cortical veins only. The clinical presentation is strongly correlated with Borden type: $2 \%$ of type I, $40 \%$ of type II and $80 \%$ of type III dural arteriovenous fistulas present with intracranial hemorrhage or neurologic deficit. ${ }^{8}$
Conservative treatment is generally recommended for patients with Borden type I fistulas. ${ }^{6}$ Endovascular approaches have become the mainstay of treatment for type II and III fistulas, but surgical treatment may also be considered when endovascular methods are not feasible or have failed. ${ }^{6}$ Excellent cure rates have been reported with transarterial, transvenous or a combination of approaches with various embolic agents such as coils and liquid embolic agents. ${ }^{6}$ In a series of 30 patients with dural arteriovenous fistulas and corticovenous reflux, complete cure was achieved in 24 patients, and and for 20 of these, cure was achieved in a single procedure with a liquid embolic agent. ${ }^{9}$

\section{Conclusion}

The diagnosis of anteriorly draining dural carotid-cavernous sinus fistulas can be difficult, because it is an uncommon cause of a red eye. Careful attention to the characteristic features of this condition (e.g., corkscrew episcleral vessels, proptosis, asymmetric intraocular pressure and chemosis) should prompt neuroimaging that includes angiography.

Given the high risk of intracranial hemorrhage in patients with cortical venous reflux, clinicians should have a high index of suspicion for dural carotid-cavernous sinus fistulas in patients with chronic red eye.

\section{References}

1. Cronau H, Kankanala RR, Mauger T. Diagnosis and management of red eye in primary care. Am Fam Physician 2010;81:137-44.

2. Miller NR. Dural carotid-cavernous fistulas: epidemiology, clinical presentation, and management. Neurosurg Clin North Am 2012;23:179-92.

3. Newton TH, Hoyt WF. Dural arteriovenous shunts in the region of the cavernous sinus. Neuroradiology 1970;1:71-81.

4. Houser OW, Campbell JK, Campbell RJ, et al. Arteriovenous malformation affecting the transverse dural sinus: an acquired lesion. Mayo Clin Proc 1979;54:651-61.

5. Moster M, Ichhpujani P. Episcleral venous pressure and glaucoma. J Curr Glaucoma Prac 2009;3:5-8.

6. Gandhi D, Chen J, Pearl M, et al. Intracranial dural arteriovenous fistulas: classification, imaging, findings, and treatment. AJNR Am J Neuroradiol 2012;33:1007-13.

7. Borden JA, Wu JK, Shucart WA. A proposed classification for spinal and cranial dural arteriovenous fistulous malformations and implications for treatment. J Neurosurg 1995;82:166-79.

8. Davies MA, TerBrugge K, Willinsky R, et al. The validity of classification for the clinical presentation of intracranial dural arteriovenous fistulas. J Neurosurg 1996;85:830-7.

9. Cognard C, Januel AC, Silva NA Jr, et al. Endovascular treatment of intracranial dural arteriovenous fistulas with cortical venous drainage: new management using Onyx. AJNR Am J Neuroradiol 2008;29:235-41.

\section{Competing interests: None declared.}

This article has been peer reviewed.

The authors have obtained patient consent.

Affiliations: Department of Ophthalmology and Vision Science (Micieli, Bedi), University of Toronto, Toronto, Ont.; Department of Neuroradiology (Krings), Toronto Western Hospital, University Health Network, Toronto, Ont.

Contributors: Jonathan Micieli and Harleen Bedi conceived the article, and all of the authors analyzed patient-specific data and information derived from the literature. All of the authors were involved in manuscript preparation, with Jonathan Micieli and Timo Krings making revisions for important intellectual content. All of the authors approved the final version for publication and agreed to act as guarantors of the work.

Correspondence to: Jonathan Micieli, jonathan.micieli@utoronto.ca 